\title{
Herbal Prescription SH003 Alleviates Docetaxel-Induced Neuropathic Pain in C57BL/6 Mice
}

\author{
Kangwook Lee, ${ }^{1}$ Jin Mo Ku, ${ }^{1}$ Yu-Jeong Choi, ${ }^{2}$ Hyun Ha Hwang, ${ }^{2}$ Miso Jeong, \\ Yun-Gyeong Kim, ${ }^{2}$ Min Jeong Kim, ${ }^{2}$ and Seong-Gyu Ko $\mathbb{D}^{1,3}$ \\ ${ }^{1}$ Institute of Safety and Effectiveness Evaluation for Korean Medicine, Seoul 02453, Republic of Korea \\ ${ }^{2}$ Department of Science in Korean Medicine, Graduate School, Kyung Hee University, Seoul 02453, Republic of Korea \\ ${ }^{3}$ Department of Preventive Medicine, College of Korean Medicine, Kyung Hee University, Seoul 02453, Republic of Korea \\ Correspondence should be addressed to Seong-Gyu Ko; epiko@khu.ac.kr
}

Received 16 April 2021; Accepted 4 August 2021; Published 11 August 2021

Academic Editor: Xia Wang

Copyright $(92021$ Kangwook Lee et al. This is an open access article distributed under the Creative Commons Attribution License, which permits unrestricted use, distribution, and reproduction in any medium, provided the original work is properly cited.

Docetaxel-based therapy has been applied to kill cancers including lung and breast cancers but frequently causes peripheral neuropathy such as mechanical allodynia. Lack of effective drugs for chemotherapy-induced peripheral neuropathy (CIPN) treatment leads us to find novel drugs. Here, we investigated whether and how novel anticancer herbal prescription SH003 alleviates mechanical allodynia in mouse model of docetaxel-induced neuropathic pain. Docetaxel-induced mechanical allodynia was evaluated using von Frey filaments. Nerve damage and degeneration in paw skin of mice were investigated by immunofluorescence staining. Neuroinflammation markers in bloodstream, lumbar (L4-L6) spinal cord, and sciatic nerves were examined by ELISA or western blot analysis. Docetaxel $(15.277 \mathrm{mg} / \mathrm{kg})$ was intravenously injected into the tail vein of C57BL/6 mice, and mechanical allodynia was followed up. SH003 $(557.569 \mathrm{mg} / \mathrm{kg})$ was orally administered at least 60 min before the mechanical allodynia test, and von Frey test was performed twice. Docetaxel injection induced mechanical allodynia, and SH003 administration restored withdrawal threshold. Meanwhile, degeneration of intraepidermal nerve fibers (IENF) was observed in docetaxel-treated mice, but SH003 treatment suppressed it. Moreover, docetaxel injection increased levels of TNF- $\alpha$ and IL-6 in plasma and expressions of phospho-NF- $\kappa$ B and phospho-STAT3 in both of lumbar spinal cord and sciatic nerves, while SH003 treatment inhibited those changes. Taken together, it is worth noting that TNF- $\alpha$ and IL- 6 in plasma and phospho-NF- $\kappa$ B and phospho-STAT3 in spinal cord and sciatic nerves are putative biomarkers of docetaxel-induced peripheral neuropathy (DIPN) in mouse models. In addition, we suggest that $\mathrm{SH} 003$ would be beneficial for alleviation of docetaxel-induced neuropathic pain.

\section{Introduction}

Cancer patients receive chemotherapy to kill malignant tumors and improve survival rate whereas it unfortunately causes severe side effects $[1,2]$. CIPN is one of the painful side effects occurring in approximately $30-70 \%$ of patients and characterized by damaged peripheral neurons [3-6]. The main symptoms include pain, muscle weakness, and muscle spasms, resulting in a decrease in the quality of life [7]. Taxanes (paclitaxel and docetaxel) are useful anticancer drugs but cause CIPN [8]. Docetaxel is one of the cytotoxic anticancer drugs and exhibits an anticancer effect by binding to tubulin, resulting in impairment of microtubule homeostasis and mitotic arrest [9]. US Food and Drug Administration (FDA) approved docetaxel as an anticancer drug against multiple types of cancers including non-smallcell lung and breast cancers $[10,11]$. Of note, docetaxel is associated with acute pain syndrome in several cancer patients [12-17]. Development of painful symptoms by DIPN may lead to discontinuation of cancer treatment regardless of therapeutic benefit of chemotherapy [18-20]. Until now, there have been no effective therapeutic options for DIPN in cancer patients [21]. Thus, this drives us to find novel medicines for treatment of DIPN.

Cancer chemotherapy typically damages the distal sensory neurons of hands or feet with severe pain [22]. 
Nonclinical studies have demonstrated that peripheral nervous system tissues, which are mainly damaged in CIPN model, include sciatic nerves, lumbar spinal cord, and dorsal root ganglion (DRG) $[23,24]$. While the mechanism underlying CIPN is still unclear, several CIPN studies have demonstrated that NF- $\kappa \mathrm{B}$ has been suggested to be one of the readouts for neuropathic pain $[8,25]$. It was reported that activation of NF- $\kappa$ B is associated with spinal cord and sciatic nerve injury in rodent model [26]. Moreover, inhibition of NF- $\kappa$ B pathway can ameliorate chronic pain in neuropathic pain rodent model. Besides NF- $\kappa$ B, STAT3 pathway has also been suggested to be involved in neuropathic pain. It has been reported that paclitaxel treatment induces neuropathic pain with increased expression of phosphorylated JAK2 and STAT3 [27]. Furthermore, another study demonstrated that chemotherapeutic agent bortezomib-induced mechanical allodynia is associated with STAT3 activation in DRG [28]. Moreover, TNF- $\alpha$-mediated activation of STAT3 plays a critical role in CIPN [29]. Therefore, activation of NF- $\kappa$ B and STAT3 can be regarded as a biomarker for CIPN. However, there is no evidence to support the fact that those biomarkers are associated with DIPN.

Novel herbal prescription $\mathrm{SH003}$ is the traditional Chinese medicine (TCM) theory-based anticancer drugs composed of Astragalus membranaceus, Angelica gigas, and Trichosanthes kirilowii Maximowicz. The anticancer effect of SH003 against breast, lung, and prostate cancer has been demonstrated through several in vitro and in vivo studies [30-38]. The safety of $\mathrm{SH} 003$ was proved by phase I clinical study for solid tumors, and phase II clinical study for wildtype EGFR lung cancer patients is in progress [39-41]. Meanwhile, phase I/II clinical study of SH003 combined with docetaxel for breast and lung cancer patients is also in progress (NCT01755923, https://clinicaltrials.gov). While it is a crucial point that TCM-based herbal medicines and supplements are the representative alternative treatment for improving health problems including cancer-related side effects [42-46], we needed to investigate whether novel herbal medicine $\mathrm{SH} 003$ alleviates docetaxel-mediated adverse effects in both nonclinical and clinical studies.

The purpose of the present study was to evaluate the mitigative effect of SH003 on DIPN in C57BL/6 mice. Intravenous injection of docetaxel decreased the withdrawal threshold of von Frey filament compared to Control group whereas oral administration of SH003 relieved this symptom. ELISA analysis showed that levels of TNF- $\alpha$ and IL- 6 in plasma were upregulated by docetaxel injection while $\mathrm{SH} 003$ treatment revered it. Docetaxel-induced upregulation of phospho-NF- $\kappa \mathrm{B}$ and phospho-STAT3 expression in sciatic nerve and spinal cord was inhibited by $\mathrm{SHO03}$ treatment. Histological analysis of mouse foot pad skin indicated that administration of $\mathrm{SH} 003$ relieved docetaxel-induced damage on peripheral nerve fibers. Therefore, the present study suggests that SH003 is applicable to alleviate DIPN.

\section{Materials and Methods}

2.1. SHOO3 and Docetaxel. $\mathrm{SH} 003$ powder was prepared as described previously. In brief, Astragalus membranaceus (333 g), Angelica gigas (333 g), and Trichosanthes kirilowii Maximowicz (333 g) were mixed and then extracted with 10 times volume of $30 \%$ ethanol at $100^{\circ} \mathrm{C}$ for $3 \mathrm{~h}$. This process was performed 2 times. The extract was dried at reduced pressure (40 Torr) at $60^{\circ} \mathrm{C}$ for $18 \mathrm{~h}$. Dried SH003 was stored at $-20^{\circ} \mathrm{C}$. Docetaxel was purchased from Sigma-Aldrich (\#01885-25MG-F, St. Louis, MO, USA). Docetaxel was dissolved in DMSO and stored at $-20^{\circ} \mathrm{C}$.

2.2. Determination of Drug Dose. No-observed-adverse-effect level (NOAEL) of SH003 determined by phase I clinical trials was $4,800 \mathrm{mg} /$ day [41]. Maximum tolerable dose (MTD) of docetaxel in cancer patients was $75 \mathrm{mg} / \mathrm{m}^{2}$ [40]. Animal equivalent dose was calculated by the following equation [47]:

$$
\text { human equivalent dose }\left(\frac{\mathrm{mg}}{\mathrm{kg}}\right)=\text { animal dose }\left(\frac{\mathrm{mg}}{\mathrm{kg}}\right) \times\left(\frac{\text { weight animal }(\mathrm{kg})}{\text { weight human }(\mathrm{kg})}\right)(1-0.75) \text {. }
$$

Human and mice weights are $65 \mathrm{~kg}$ and $0.02 \mathrm{~kg}$, respectively. Since there is an individual difference in metabolic rate, exponent for body surface area is 0.75 . Thus, animal equivalent dose of $\mathrm{SH} 003$ and docetaxel was determined as $557.569 \mathrm{mg} / \mathrm{kg}$ and $15.277 \mathrm{mg} / \mathrm{kg}$, respectively.

\subsection{Docetaxel-Induced Neuropathy Mouse Model and Ex-} periment Schedule. All procedures in animal experiments were approved by Kyung Hee University Institutional Animal Care and Use Committee (KHU-IACUC; KHSASP-19322). C57BL/6N mice (5 weeks old) were purchased from Nara Biotech (Seoul, South Korea). Mice were housed under constant temperature $\left(24 \pm 2^{\circ} \mathrm{C}\right)$ with light-dark cycle, and food and water were freely available. Two experiments were performed. The timelines of experiment " 1 " and experiment "2" are shown in Figure 1.

In experiment " 1 ," mice were randomly divided into different treatment groups as follows: Control $(n=6)$ and Docetaxel $(n=6)$. Before injection of docetaxel on the $1^{\text {st }}$ day, baseline withdrawal threshold in each mouse was determined. Mice were treated with a single intravenous injection of docetaxel to model DIPN, and neuropathic symptom was followed up.

In experiment " 2 ," mice were randomly divided into different groups as follows: "Control" $(n=5)$, "Docetaxel" $(n=5)$, "Docetaxel + SH003" $(n=5)$. After determination of baseline withdrawal threshold by von Frey test on the $1^{\text {st }}$ day, 

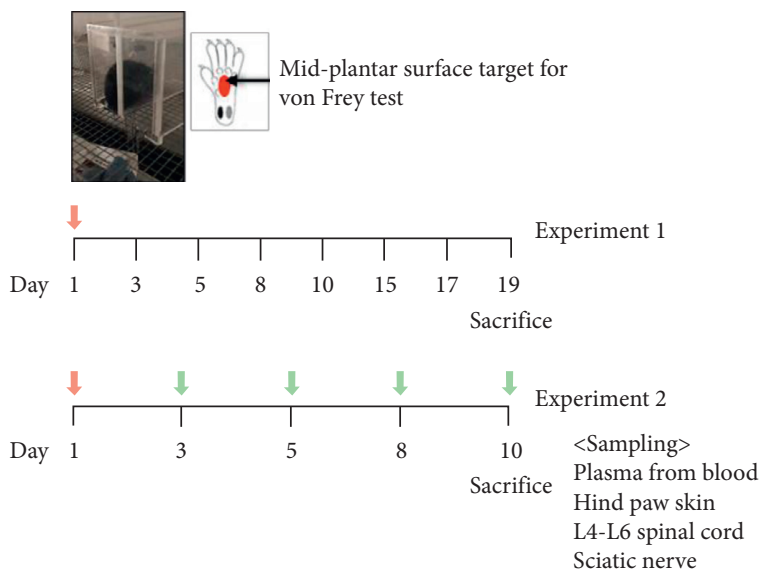

$\Longrightarrow$ Docetaxel injection

$\rightarrow$ SH003 administration

Figure 1: Timelines of the in vivo experimental design.

mice in "Docetaxel" and "Docetaxel + SH003" were intravenously injected with $50 \mu \mathrm{L}$ docetaxel while mice in "Control" were injected with the same volume of DMSO, which did not exceed $5 \mathrm{~mL} / \mathrm{kg}$ according to guidelines [48]. SH003 was orally administered at least $60 \mathrm{~min}$ before the performance of mechanical allodynia test. SH003 administration and von Frey filament test were performed 2-3 times a week. At the end of the experiment, mice were euthanized by $100 \%$ carbon dioxide in chamber by $30-70 \% / \mathrm{min}$ filling rate, followed by cervical dislocation. The hind paw skin of mice was collected for staining of IENF. For evaluation of the inflammatory damage on peripheral neurons, lumbar (L4-L6) spinal cord and both left and right sciatic nerves were collected.

2.4. Measurement of Mechanical Allodynia. Mechanical allodynia was evaluated using von Frey filaments (JD-SI$11 F$, Jeungdo B and P, Seoul, South Korea) according to standard operating procedure from the Jackson Laboratory mouse neurobehavioral phenotyping facility. In brief, the room atmosphere was maintained with constant temperature $\left(24 \pm 2^{\circ} \mathrm{C}\right)$, humidity $(50 \pm 20 \%)$, and lighting (400-500 lux) without noise. Prior to the start of von Frey test, mice were individually placed on stainless steel mesh floor with acrylic cover $\left(\mathrm{L}^{*} \mathrm{~W}^{*} \mathrm{H}=10^{*} 8^{*} 7 \mathrm{~cm}\right)$ and left without disturbance for a minimum of $30 \mathrm{~min}$ to acclimate. The midplantar surface of left hind paw of mice was poked with filament $(0.4 \mathrm{~g})$ for 3 seconds until it bends, and each application was presented at least 3 times. If there was no response with the starting filament $(0.4 \mathrm{~g})$, it was changed to the next highest filament $(0.6 \mathrm{~g})$ in the series and continuously moved through the series in order until a withdrawal response was observed or the $2 \mathrm{~g}$ filament was presented. If there was response with the starting filament, it was moved to the next lowest filament in the series until a withdrawal response was not observed or the $0.02 \mathrm{~g}$ filament was presented. Licking and shaking of the affected paw were determined as the withdrawal responses. The whole procedure from acclimation to determination of withdrawal responses was repeated once again. Mechanical allodynia threshold values from two independent experiments were recorded and averaged in each group.

2.5. Immunofluorescence Staining of IENF. The hind paw skin was isolated from all mice and immediately fixed with $4 \%$ paraformaldehyde for $6 \mathrm{~h}$. Fixed skin samples were dehydrated using $30 \%$ sucrose solution for $16 \mathrm{~h}$. For the cryosection, dehydrated tissues were embedded in OCT compound and frozen at liquid nitrogen. Frozen sections of $20 \mu \mathrm{m}$ were stained with anti-PGP9.5 antibody $(1: 800$, ab8189, Abcam, Cambridge, UK) and IgG secondary antibody conjugated with Alexa Fluor 488 (1:2,000, A28175, Invitrogen Co., Carlsbad, CA, USA). The images were acquired using Zeiss LSM 5 PASCAL confocal laser scanning microscope system (Carl Zeiss AG, Oberkochen, Germany) at a magnification of $10 \mathrm{x}$.

2.6. Enzyme-Linked Immunosorbent Assay (ELISA). Levels of inflammatory cytokines TNF-a and IL- 6 were assessed using a DuoSet ELISA kit (555268 and 555240, respectively, BD Biosciences, San Diego, USA) according to the manufacturer's instructions. In brief, the whole blood was collected from left ventricle and transferred to EDTA tube (367835, BD Biosciences, San Diego, USA). After centrifugation at 2,000 rpm for $10 \mathrm{~min}$, plasma from blood was prepared and stored in a deep freezer at $-80^{\circ} \mathrm{C}$ until use. 96-well plates were coated with capture antibody in ELISA coating buffer and incubated overnight at $4^{\circ} \mathrm{C}$. The plates were then washed with wash buffer and subsequently blocked with assay diluent at RT for $1 \mathrm{~h}$. Diluted standard and plasma samples were added to plates and incubated at RT for $2 \mathrm{~h}$. Detection antibody and streptavidin-conjugated horseradish peroxidase were added to the plates, followed by incubation at RT for $1 \mathrm{~h}$. Tetramethylbenzidine substrate was added and incubated at RT for $0.5 \mathrm{~h}$. The reaction was ended by adding stop buffer. The optical density was measured at $450 \mathrm{~nm}$ on an automated ELISA reader (Versa Max, Molecular Devices, CA, USA).

2.7. Western Blot Analysis. Total protein from lumbar (L4L6) spinal cord and sciatic nerves were extracted using RIPA buffer (R2002, Biosesang, Gyeonggi-do, South Korea) with protease and phosphatase inhibitors. The same amount of proteins was quantified by Bradford protein assay (Bio-Rad, Hercules, CA, USA). The proteins were separated by 8 or $15 \%$ SDS-PAGE, followed by transfer to nitrocellulose membranes. After blocking with blocking buffer including $1 \%(\mathrm{w} / \mathrm{v})$ skim milk and $1 \%(\mathrm{w} / \mathrm{v})$ BSA at RT for 1 hour, the membrane was incubated with anti-NF- $\mathrm{B}$ (\#8242), antiphospho-NF-кB (\#3033), anti-STAT3 (\#4904), anti-phospho-STAT3 (\#9145), and anti-GAPDH (\#5174) at $4^{\circ} \mathrm{C}$ for 16-24h. All antibodies were purchased from Cell Signaling Technology (Beverly, MA, USA). Horseradish peroxidase(HRP-) conjugated secondary IgG antibodies (Calbiochem, San Diego, CA, USA) were further incubated with the 
membrane at RT for $1 \mathrm{~h}$. An enhanced chemiluminescence kit (DG-WP100, DoGen, Seoul, South Korea) was used for detection of HRP signal.

2.8. Statistical Analysis. Statistical analysis was performed using Prism (GraphPad, San Diego, CA, USA). The differences of means between the groups were analyzed by oneway or two-way ANOVA using Tukey's or Sidak's multiple comparisons test, respectively. $P$ value $<0.05$ means statistically significant difference. Results were represented as mean \pm standard deviation (SD) or standard error of the mean (SEM).

\section{Results and Discussion}

3.1. Intravenous Injection of DIPN in C57BL/6 Mice. Docetaxel is a well-known anticancer drug with severe side effects including CIPN with pain in the hands and feet $[49,50]$. The present study was performed to model DIPN. Docetaxel was intravenously injected into mice tail vein, and mechanical allodynia threshold by von Frey test was followed up. On the $5^{\text {th }}$ day, mechanical withdrawal threshold of Docetaxel group began to be lower than that of Control group (Figure $2(\mathrm{a})$ ). On the $10^{\text {th }}$ day, the biggest difference of mechanical allodynia threshold between Control group and Docetaxel group occurred, while there were no differences after the $15^{\text {th }}$ day (Figure 2(a)). Drug treatment did not affect body weights of mice until 19 days (Figure 2(b)). Therefore, we determined to perform further efficacy study until the $10^{\text {th }}$ day. It is common for taxane-based adjuvant chemotherapy to induce acute pain syndrome [51]. In fact, the incidence of taxane acute pain syndrome is more common with docetaxel than paclitaxel whereas docetaxel triggers less peripheral neuropathy than paclitaxel $[16,52]$. Docetaxelmediated pain flare occurs within 4-5 days after docetaxel chemotherapy and lasts about 4 days [52]. Consistent with clinical findings, in vivo model for docetaxel-related acute pain syndrome was successfully developed by a single intravenous injection of docetaxel at MTD.

3.2. Oral Administration of SHOO3 Mitigated Docetaxel-Induced Mechanical Allodynia at Hind Paws of C57BL/6 Mice. Next, we further examined whether SH003 mitigates docetaxel-induced mechanical allodynia. Although mechanical threshold of "Docetaxel" was higher than that of "Docetaxel + SH003" on the $3^{\text {rd }}$ day, the threshold value of two groups was not statistically different with "Control." While docetaxel treatment induced mechanical allodynia, SH003 treatment relieved it on the $8^{\text {th }}$ and $10^{\text {th }}$ day (Figure 3(a)). Drug treatment did not affect body weights of mice until 10 days (Figure 3(b)). The data showed that SH003 administration mitigated DIPN. SH003 is a TCMbased novel herbal mixture for treating several malignant cancers including breast, prostate, and lung cancers. Anticancer effect and mode of action have been clearly demonstrated by numerous nonclinical studies [30-38]. In fact, conventional chemodrugs have adverse side effects because of their cytotoxicity to healthy cells as well as cancer cells regardless of therapeutic benefit. Our previous study reported that $\mathrm{SH} 003$ is safe in rats according to toxicity studies with Good Laboratory Practice (GLP) regulations [36]. Oral administration of $\mathrm{SH} 003$ at various doses (500; 1,000; $2,000 \mathrm{mg} / \mathrm{kg}$ ) did not show any problem in mortality, food intake, haematological value, organ weight, histopathology, etc. In sum, we suggest that SH003 is safe and effective herbal medicine for treatment of DIPN as well as cancer.

3.3. SHOO3 Administration Inhibited Docetaxel-Induced Reduction of IENF at Hind Paws of C57BL/6 Mice. IENF are bare nerve endings within junction between dermis and epidermis and play a role in transmission of peripheral pain [53, 54]. Clinically, chemotherapy damages distal IENF, resulting in loss of IENF in hands and feet in patients with CIPN. [55-59]. In the rodent CIPN model, degeneration of IENF in paw skin is detected although the contribution of IENF loss to the pathobiology of CIPN is not fully elucidated [60-62]. Of note, chemotherapeutic drugs including paclitaxel and oxaliplatin induce loss of IENF with severe pain in rodent model while prevention of IENF loss inhibits neuropathic pain $[53,55,63]$. Thus, the reduction of IENF loss could be regarded as one of therapeutic markers in CIPN model. We further investigated the therapeutic effect of SH003 on IENF degeneration in mice paw skin. Compared to Control, IENF seem to be fragmented and degenerated (white arrow) in docetaxel-treated mice, whose morphological changes indicate axonal degeneration (Figures 4(a) and 4(b)). SH003 treatment decreased docetaxel-induced histopathological changes whereas a layer (yellow arrow) was thickened (Figure 4(c)). This layer located in the mid-epidermis is stratum lucidum containing dead keratinocytes and is present in the palms and soles [64]. The thickness of this layer is regulated by the rate of mitosis of the epidermal cells [65]. Stratum lucidum layer contains a large amount of eleidin which is known to protect skins by blocking infiltration of water [66]. However, the role of stratum lucidum in neuropathic pain is unknown yet. Although further studies are still needed to investigate the possible role of components in stratum lucidum in DIPN model, our data suggest that histological change in stratum lucidum is one of the therapeutic markers for SH003 on DIPN.

3.4. Oral Administration of SHOO3 Inhibited Docetaxel-Induced Production of Proinflammatory Cytokines in Bloodstream of C57BL/6 Mice. In CIPN model, pain mediators including inflammatory cytokines are increased in peripheral nerve spinal cord and sciatic nerves [67]. In vivo studies demonstrated that levels of TNF- $\alpha$ and IL- 6 are elevated in CIPN animal model $[68,69]$. It was also demonstrated that neutralizing antibodies against TNF- $\alpha$ or IL- 6 reduce chemotherapy-induced allodynia $[69,70]$. The present study investigated whether SH003 reduces TNF- $\alpha$ and IL-6 in DIPN model. Docetaxel injection increased levels of TNF- $\alpha$ and IL-6 in plasma sample (Figures 5(a) and 5(b)). The present data showed that DIPN is associated with inflammatory neuropathy [8]. In SH003 group, the inflammatory 


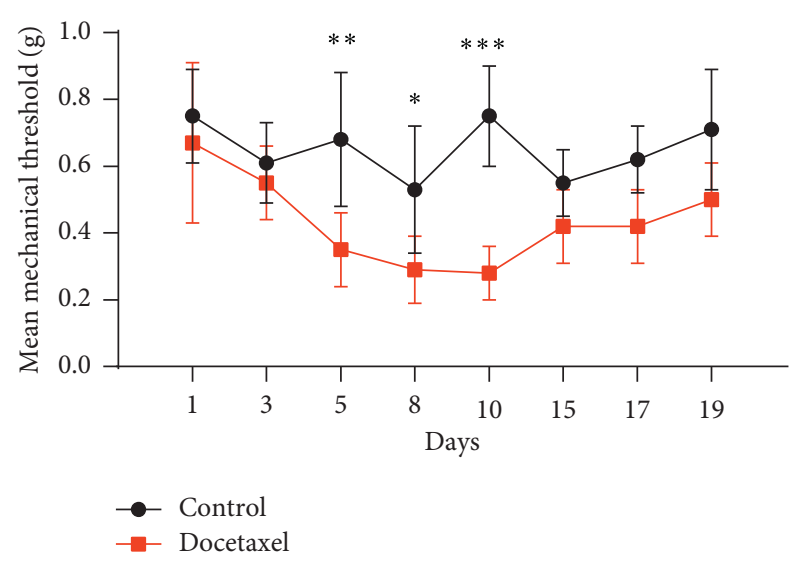

(a)

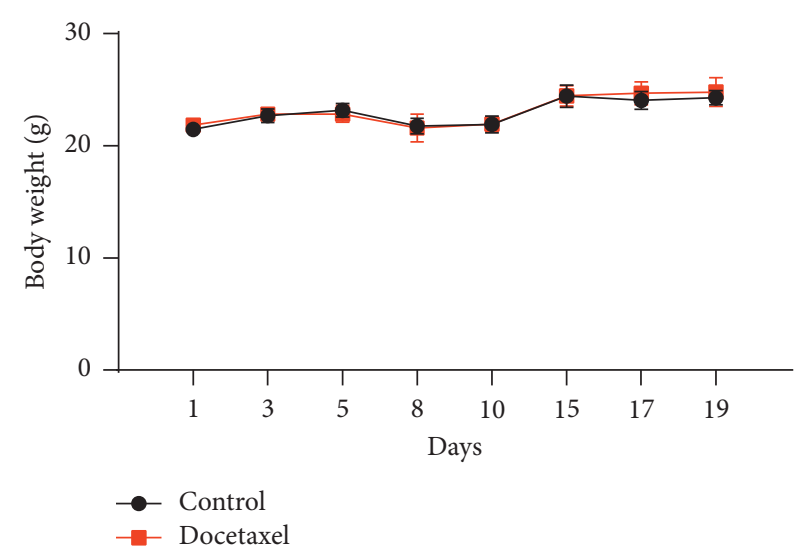

(b)

Figure 2: Docetaxel injection induced mechanical allodynia in C57BL/6 mice. The mice were divided into "Control" ( $n=6)$ and "Docetaxel" $(n=6)$. Docetaxel was intravenously injected via tail vein of C57BL/6 mice on the $1^{\text {st }}$ day, and mechanical allodynia threshold by von Frey filament was followed up. Mean mechanical threshold (a) and body weight (b). The differences of means between the groups were analyzed by two-way ANOVA using Sidak's multiple comparisons test $\left({ }^{*} P<0.05,{ }^{* *} P<0.01\right.$, and ${ }^{* * *} P<0.001$; Docetaxel vs. Control).

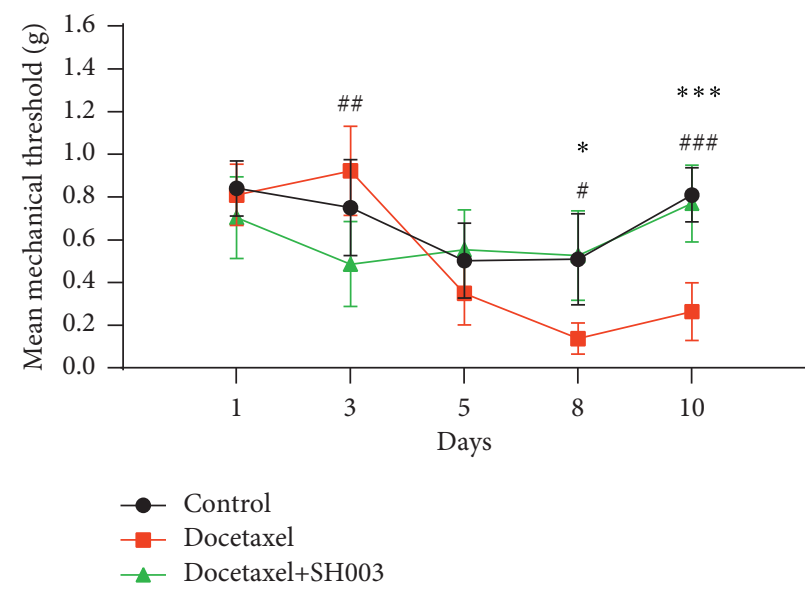

(a)

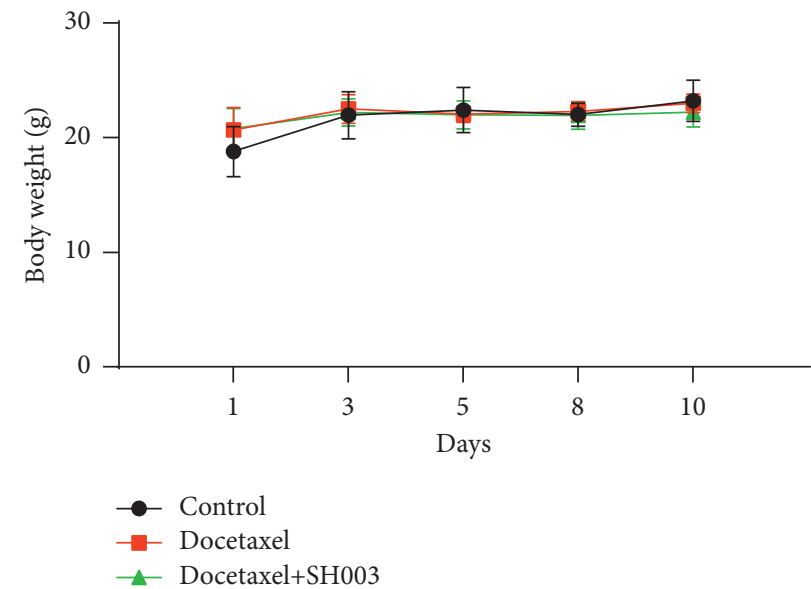

(b)

FIGURE 3: Effect of SH003 administration on docetaxel-induced mechanical allodynia in C57BL/6 mice. The mice were divided into Control $(n=5)$, Docetaxel $(n=5)$, and Docetaxel $+\mathrm{SH} 003(n=5)$ groups. Docetaxel was intravenously injected via tail vein of C57BL/6 mice on the $1^{\text {st }}$ day, and mechanical allodynia threshold by von Frey filament was followed up. From $3^{\text {rd }}$ day, SH003 was orally administered at least $60 \mathrm{~min}$ before the performance of mechanical allodynia test. Mean mechanical threshold (a) and body weight (b). Data are presented as mean \pm SEM. The differences of means between the groups were analyzed by two-way ANOVA using Sidak's multiple comparisons test $\left({ }^{*} P<0.05\right.$, Docetaxel vs. Control; ${ }^{* * *} P<0.001$, Docetaxel vs. Control; ${ }^{\#} P<0.05$, Docetaxel + SH003 vs. Docetaxel; ${ }^{\# \#} P<0.001$, Docetaxel + SH003 vs. Docetaxel).

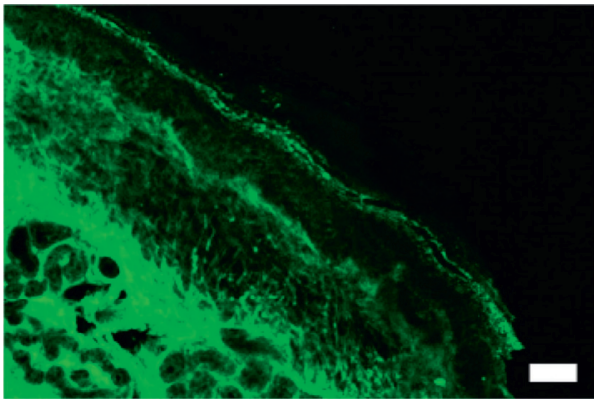

(a)

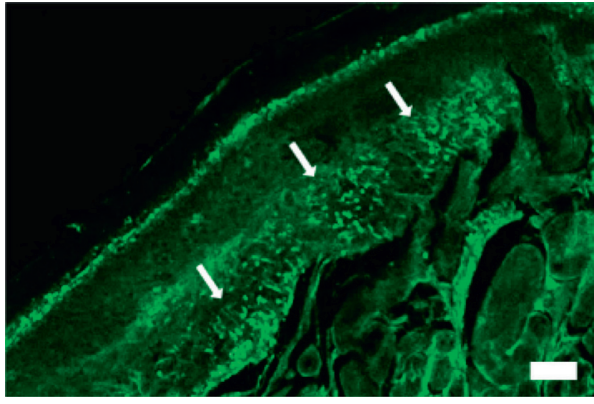

(b)

Figure 4: Continued. 


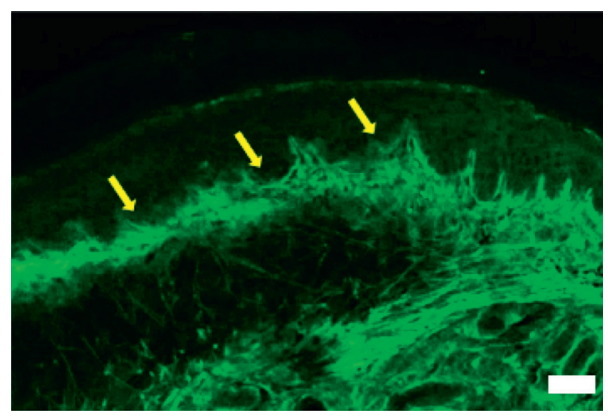

(c)

FIGURE 4: Effect of SH003 administration on degeneration of IENF induced by docetaxel. The hind paw skins were isolated and prepared for immunofluorescence staining of IENF in Control (a), Docetaxel (b), and Docetaxel + SH003 (c) groups. The representative images of PGP 9.5-labeled IENF in paw skins were acquired using Zeiss LSM5 PASCAL confocal laser scanning microscope system. White scale bar indicates $50 \mu \mathrm{m}$ (magnification: 10x). White and yellow arrows indicate the degenerated IENF and stratum lucidum layer, respectively.

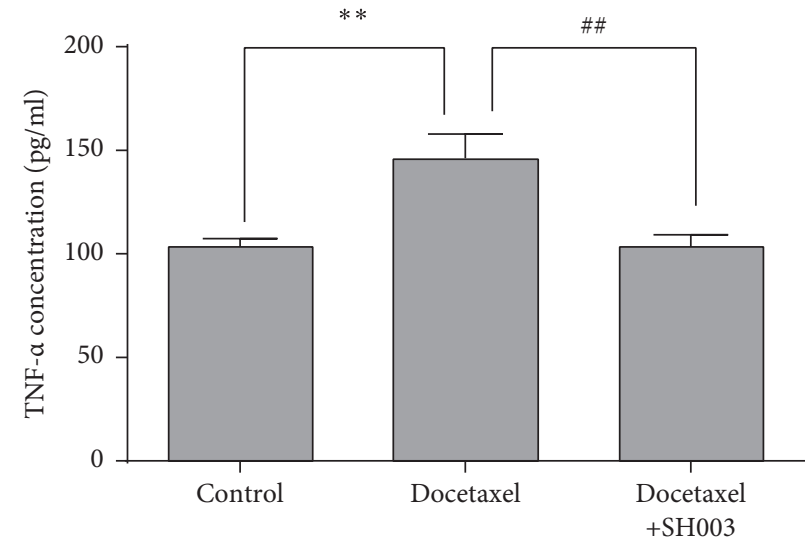

(a)

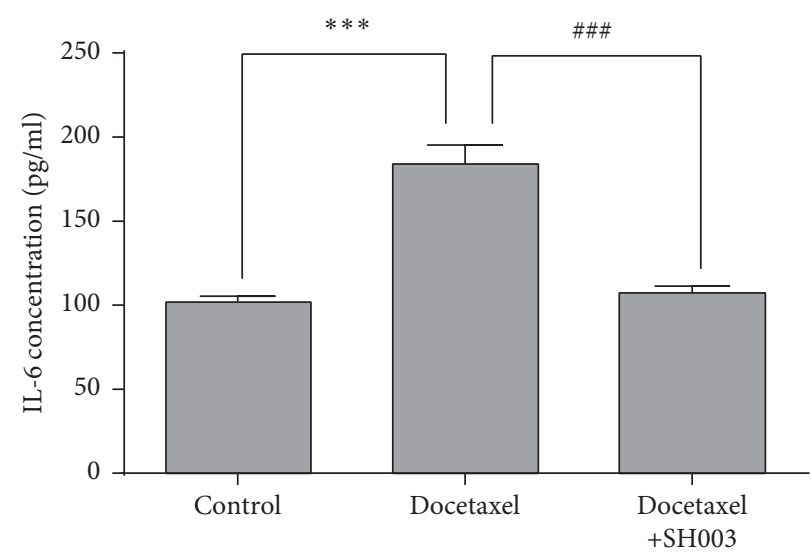

(b)

FIGURE 5: Effect of SH003 administration on the expression of TNF- $\alpha$ and IL-6 in plasma of DIPN mice. Plasma sample was prepared, and levels of inflammatory cytokines TNF- $\alpha$ (a) and IL-6 (b) were assessed using a DuoSet ELISA kit according to the manufacturer's instructions. Data are presented as mean \pm SD of the mean ( $n=5$ for each group). The differences of means between the groups were analyzed by one-way ANOVA using Tukey's post hoc test $\left({ }^{* *} P<0.001\right.$, Docetaxel $v$ s. Control; ${ }^{* * *} P<0.001$, Docetaxel $v$ s. Control; ${ }^{\# \#} P<0.01$, Docetaxel + SH003 vs. Docetaxel; ${ }^{\# \# \# ~} P<0.001$, Docetaxel + SH003 vs. Docetaxel).

level increased by docetaxel was reduced as much as Control group (Figures 5(a) and 5(b)). We also demonstrated that SH003 inhibition of DIPN is mediated by relieving inflammation.

\subsection{Oral Administration of SHOO3 Inhibited Docetaxel-In-} duced Phosphorylation of NF- $\kappa B$ and STAT3 in Both L4-L6 Spinal Cord and Sciatic Nerve of C57BL/6 Mice. NF- $\kappa \mathrm{B}$ and STAT3 would be one of the readouts for CIPN [8, 25-29]. Moreover, TNF- $\alpha$ and IL- 6 are involved in the activation of $\mathrm{NF}-\kappa \mathrm{B}$ and STAT3 in neuroinflammation [71-73]. Thus, we further examined whether $\mathrm{SH} 003$ treatment inhibits NF- $\kappa$ B and STAT3 in both of lumbar spinal cord and sciatic nerves. Docetaxel treatment increased the expression of phospho-NF- $\kappa$ B and phospho-STAT3 in both L4-L6 spinal cords and sciatic nerves whereas $\mathrm{SH} 003$ treatment reversed it (Figures 6(a) and 6(b)). The present study suggested that activation of NF- $\kappa$ B and STAT3 is a putative biomarker of DIPN while further studies are necessary to decipher how they are involved in the development and progression of DIPN. Moreover, SH003 inhibition of NF- $\kappa$ B and STAT3 shown in our data would be a hint for $\mathrm{SH} 003$ application to other peripheral neuropathic diseases. Deregulation of NF$\kappa \mathrm{B}$ and STAT3 appears to be involved in the progression of neuropathic pain in diabetes [74-77]. Alcoholic neuropathy is tightly linked to NF- $\kappa \mathrm{B}$ deregulation $[78,79]$. NF- $\kappa \mathrm{B}$ deregulation is likely to be related to dysimmune neuropathies such as acute Guillain-Barré syndrome [80]. Therefore, SH003 may be applicable for treating other neuropathic diseases related to the deregulation of NF- $\kappa \mathrm{B}$ and STAT3. 


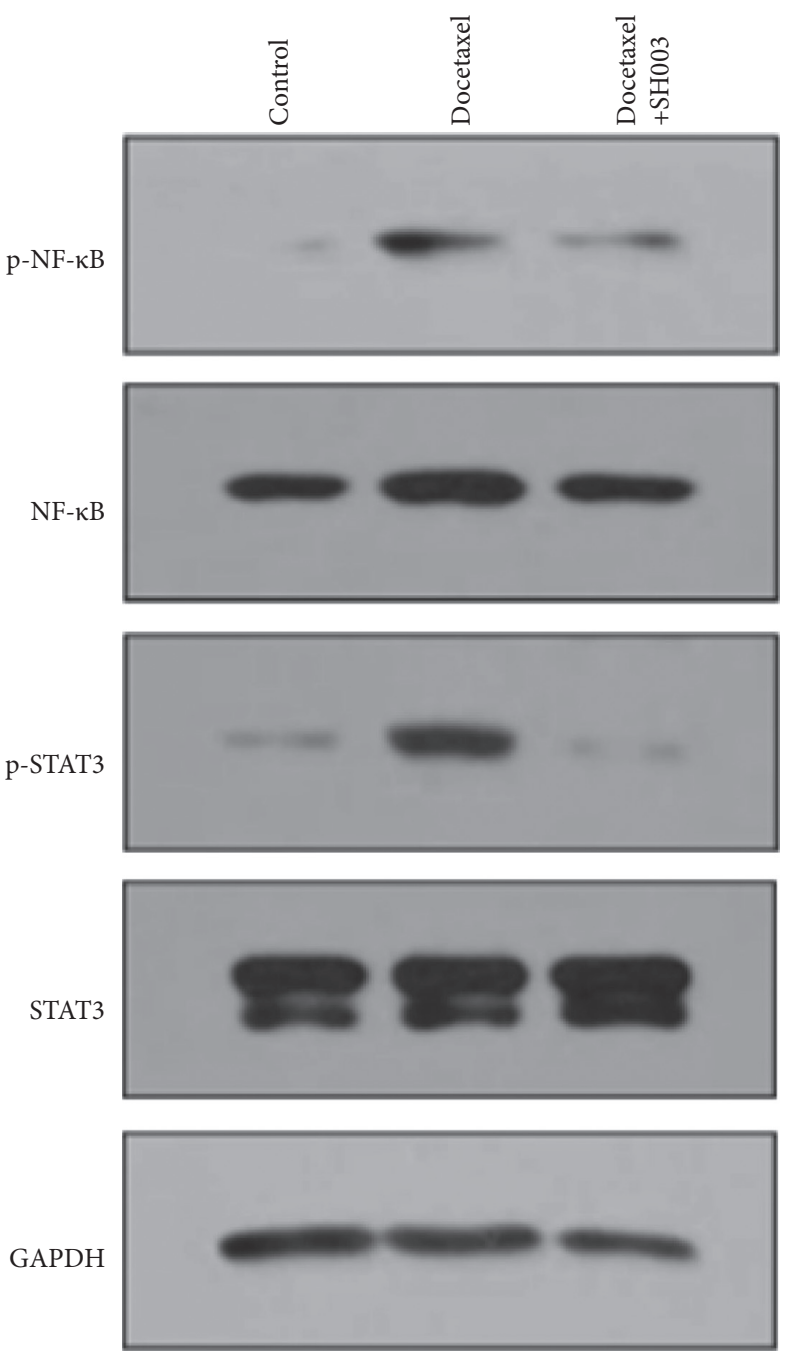

(a)
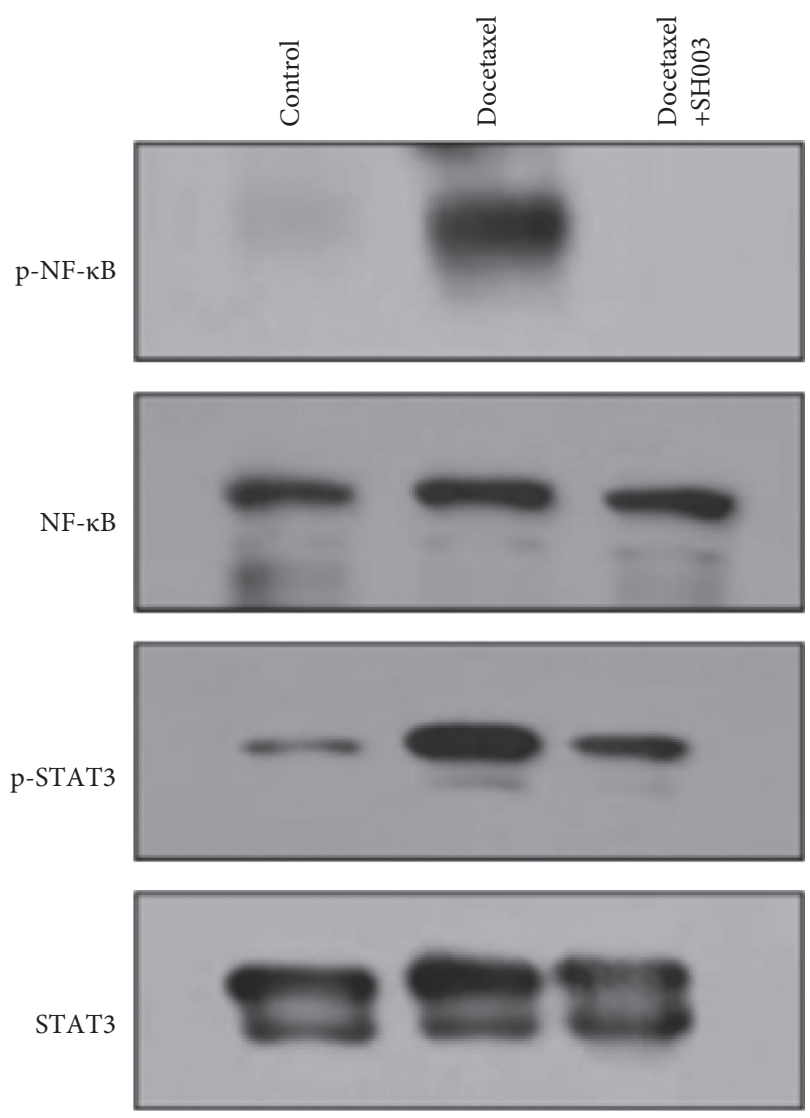

GAPDH

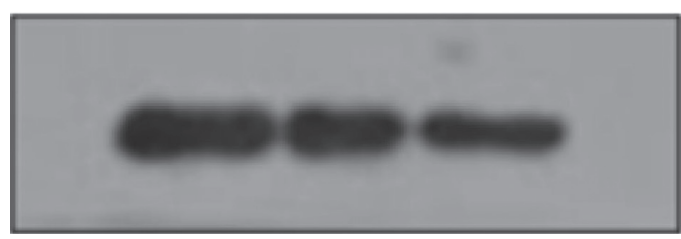

(b)

FIGURE 6: Effect of SH003 administration on the expression of NF- $\kappa$ B and STAT3 in lumbar (L4-L6) spinal cord and sciatic nerves of DIPN mice. At the end of the experiment, lumbar (L4-L6) spinal cord and sciatic nerves were isolated. Total proteins were extracted and separated by SDS-PAGE. Expression of NF- $\kappa$ B and STAT3 from spinal cord (a) and sciatic nerves (b) was analyzed by western blotting. GAPDH was used as internal marker.

\section{Conclusions}

Single injection of docetaxel at MTD can develop acute pain syndrome model in C57BL/6 mice. Moreover, activation of $\mathrm{NF}-\kappa \mathrm{B}$ and STAT3 in sciatic nerves and L4-L6 spinal cords and upregulation of TNF- $\alpha$ or IL- 6 in bloodstream may be biomarkers of DIPN in mouse model. However, further studies are needed to investigate the role of those putative biomarkers in the development and progression of DIPN. Furthermore, we suggest that $\mathrm{SH} 003$ administration is able to relieve docetaxel-induced neuropathic pain and this efficacy needs to be monitored in current clinical studies.

\section{Data Availability}

All datasets used and/or analyzed during the current study are available from the corresponding author on reasonable request.

\section{Conflicts of Interest}

The authors declare that there are no conflicts of interest regarding the publication of this study.

\section{Acknowledgments}

This research was supported by a grant from the Korean Medicine R\&D Project of the Ministry of Health and Welfare (HI18C2382) and the National Research Foundation of Korea (NRF) grant funded by the Korean government (MSIT) (no. 2020R1A5A201941311).

\section{References}

[1] C. Maihofner, I. Diel, H. Tesch, T. Quandel, and R. Baron, "Chemotherapy-induced peripheral neuropathy (CIPN): current therapies and topical treatment option with high- 
concentration capsaicin," Supportive Care in Cancer, vol. 29, 2021.

[2] B. Fu, N. Wang, H.-Y. Tan, S. Li, F. Cheung, and Y. Feng, "Multi-component herbal products in the prevention and treatment of chemotherapy-associated toxicity and side effects: a review on experimental and clinical evidences," Frontiers in Pharmacology, vol. 9, p. 1394, 2018.

[3] M. Seretny, G. L. Currie, E. S. Sena et al., "Incidence, prevalence, and predictors of chemotherapy-induced peripheral neuropathy: a systematic review and meta-analysis," Pain, vol. 155, no. 12, pp. 2461-2470, 2014.

[4] L. A. Colvin, "Chemotherapy-induced peripheral neuropathy: where are we now?" Pain, vol. 160, no. 1, pp. S1-S10, 2019.

[5] T. Bao, O. Goloubeva, C. Pelser et al., "A pilot study of acupuncture in treating bortezomib-induced peripheral neuropathy in patients with multiple myeloma," Integrative Cancer Therapies, vol. 13, no. 5, pp. 396-404, 2014.

[6] J. E. Cunningham, T. Kelechi, K. Sterba, N. Barthelemy, P. Falkowski, and S. H. Chin, "Case report of a patient with chemotherapy-induced peripheral neuropathy treated with manual therapy (massage)," Supportive Care in Cancer, vol. 19, no. 9, pp. 1473-1476, 2011.

[7] C. Brami, T. Bao, and G. Deng, "Natural products and complementary therapies for chemotherapy-induced peripheral neuropathy: a systematic review," Critical Reviews in Oncology, vol. 98, pp. 325-334, 2016.

[8] R. Zajaczkowska, M. Kocot-Kepska, W. Leppert, A. Wrzosek, J. Mika, and J. Wordliczek, "Mechanisms of chemotherapyinduced peripheral neuropathy," International Journal of Molecular Sciences, vol. 20, no. 6, 2019.

[9] E. Mukhtar, V. M. Adhami, and H. Mukhtar, "Targeting microtubules by natural agents for cancer therapy," Molecular Cancer Therapeutics, vol. 13, no. 2, pp. 275-284, 2014.

[10] F. K. Engels, A. Sparreboom, R. A. A. Mathot, and J. Verweij, "Potential for improvement of docetaxel-based chemotherapy: a pharmacological review," British Journal of Cancer, vol. 93, no. 2, pp. 173-177, 2005.

[11] A. Montero, F. Fossella, G. Hortobagyi, and V. Valero, "Docetaxel for treatment of solid tumours: a systematic review of clinical data," The Lancet Oncology, vol. 6, no. 4, pp. 229-239, 2005.

[12] R. Fazio, A. Quattrini, A. Bolognesi et al., "Docetaxel neuropathy: a distal axonopathy," Acta Neuropathologica, vol. 98, no. 6, pp. 651-653, 1999.

[13] Y.-N. Chan, Y.-W. Jheng, P.-J. Wang, C.-Y. Chen, M.-W. Lin, and Y.-J. Wang, "Taxane-induced peripheral neuropathy: objective and subjective comparison between paclitaxel and docetaxel in patients with breast cancer," Clinical Journal of Oncology Nursing, vol. 23, no. 5, pp. 494-501, 2019.

[14] C. Tofthagen, R. D. McAllister, and C. Visovsky, "Peripheral neuropathy caused by Paclitaxel and docetaxel: an evaluation and comparison of symptoms," Journal of the advanced practitioner in oncology, vol. 4, no. 4, pp. 204-215, 2013.

[15] L. Eckhoff, A. S. Knoop, M.-B. Jensen, B. Ejlertsen, and M. Ewertz, "Risk of docetaxel-induced peripheral neuropathy among 1,725 Danish patients with early stage breast cancer," Breast Cancer Research and Treatment, vol. 142, no. 1, pp. 109-118, 2013.

[16] R. Fernandes, S. Mazzarello, B. Hutton et al., "Taxane acute pain syndrome (TAPS) in patients receiving taxane-based chemotherapy for breast cancer-a systematic review," Supportive Care in Cancer, vol. 24, no. 8, pp. 3633-3650, 2016.
[17] N. Chiu, L. Zhang, R. Dent et al., "A prospective study of docetaxel-associated pain syndrome," Supportive Care in Cancer, vol. 26, no. 1, pp. 203-211, 2018.

[18] J. Verweij, M. Clavel, and B. Chevalier, "Paclitaxel (TaxolTM) and docetaxel (TaxotereTM): not simply two of a kind," Annals of Oncology, vol. 5, no. 6, pp. 495-505, 1994.

[19] I. Gilron, J. M. Bailey, D. Tu, R. R. Holden, D. F. Weaver, and R. L. Houlden, "Morphine, gabapentin, or their combination for neuropathic pain," New England Journal of Medicine, vol. 352, no. 13, pp. 1324-1334, 2005.

[20] M. Ewertz, C. Qvortrup, and L. Eckhoff, "Chemotherapyinduced peripheral neuropathy in patients treated with taxanes and platinum derivatives," Acta Oncologica, vol. 54, no. 5, pp. 587-591, 2015.

[21] C. C. Henke, J. Cabri, L. Fricke et al., "Strength and endurance training in the treatment of lung cancer patients in stages IIIA/IIIB/IV," Supportive Care in Cancer, vol. 22, no. 1, pp. 95-101, 2014.

[22] M. Sisignano, R. Baron, K. Scholich, and G. Geisslinger, "Mechanism-based treatment for chemotherapy-induced peripheral neuropathic pain," Nature Reviews Neurology, vol. 10, no. 12, pp. 694-707, 2014.

[23] Y. Han and M. T. Smith, "Pathobiology of cancer chemotherapy-induced peripheral neuropathy (CIPN)," Frontiers in Pharmacology, vol. 4, p. 156, 2013.

[24] A. Hoke and M. Ray, "Rodent models of chemotherapy-induced peripheral neuropathy," ILAR Journal, vol. 54, no. 3, pp. 273-281, 2014.

[25] A. S. Ahmed, S. Berg, K. Alkass et al., "NF-kappaB-Associated pain-related neuropeptide expression in patients with degenerative disc disease," International Journal of Molecular Sciences, vol. 20, no. 3, 2019.

[26] W. Ma and M. A. Bisby, "Increased activation of nuclear factor kappa B in rat lumbar dorsal root ganglion neurons following partial sciatic nerve injuries," Brain Research, vol. 797, no. 2, pp. 243-254, 1998.

[27] L. Brandolini, E. Benedetti, P. A. Ruffini et al., "CXCR1/2 pathways in paclitaxel-induced neuropathic pain," Oncotarget, vol. 8, no. 14, pp. 23188-23201, 2017.

[28] C.-C. Liu, Z.-X. Huang, X. Li et al., "Upregulation of NLRP3 via STAT3-dependent histone acetylation contributes to painful neuropathy induced by bortezomib," Experimental Neurology, vol. 302, pp. 104-111, 2018.

[29] Y. Y. Li, H. Li, Z. L. Liu et al., “Activation of STAT3-mediated CXCL12 up-regulation in the dorsal root ganglion contributes to oxaliplatin-induced chronic pain," Molecular Pain, vol. 13, Article ID 1744806917747425, 2017.

[30] Y. K. Choi, S. G. Cho, S. M. Woo et al., "Herbal extract SH003 suppresses tumor growth and metastasis of MDA-MB-231 breast cancer cells by inhibiting STAT3-IL-6 signaling," Mediators of Inflammation, vol. 2014, Article ID 492173, 2014.

[31] E. K. Choi, S.-M. Kim, S.-W. Hong et al., "SH003 selectively induces p73-dependent apoptosis in triple-negative breast cancer cells," Molecular Medicine Reports, vol. 14, no. 4, pp. 3955-3960, 2016.

[32] H. S. Choi, M. K. Kim, K. Lee et al., "SH003 represses tumor angiogenesis by blocking VEGF binding to VEGFR2," Oncotarget, vol. 7, no. 22, pp. 32969-32979, 2016.

[33] Y.-J. Choi, Y. K. Choi, K. M. Lee, S.-G. Cho, S.-Y. Kang, and S.-G. Ko, "SH003 induces apoptosis of DU145 prostate cancer cells by inhibiting ERK-involved pathway," BMC Complementary and Alternative Medicine, vol. 16, no. 1, p. 507, 2016. 
[34] S.-M. Woo, A. J. Kim, Y. K. Choi, Y. C. Shin, S.-G. Cho, and S.-G. Ko, "Synergistic effect of SH003 and doxorubicin in triple-negative breast cancer," Phytotherapy Research, vol. 30, no. 11, pp. 1817-1823, 2016.

[35] H. S. Choi, S. G. Cho, M. K. Kim et al., "SH003 enhances paclitaxel chemosensitivity in MCF-7/PAX breast cancer cells through inhibition of MDR1 activity," Molecular and Cellular Biochemistry, vol. 426, no. 1-2, pp. 1-8, 2017.

[36] Y. K. Choi, S.-G. Cho, Y.-J. Choi et al., "SH003 suppresses breast cancer growth by accumulating p62 in autolysosomes," Oncotarget, vol. 8, no. 51, pp. 88386-88400, 2017.

[37] H. S. Seo, J. M. Ku, H. J. Lee et al., "SH003 reverses drug resistance by blocking signal transducer and activator of transcription 3 (STAT3) signaling in breast cancer cells," Bioscience Reports, vol. 37, no. 6, 2017.

[38] T. W. Kim, C. Cheon, and S.-G. Ko, "SH003 activates autophagic cell death by activating ATF4 and inhibiting G9a under hypoxia in gastric cancer cells," Cell Death \& Disease, vol. 11, no. 8, p. 717, 2020.

[39] C. Cheon, S. Kang, Y. Ko et al., "Single-arm, open-label, doseescalation phase I study to evaluate the safety of a herbal medicine SH003 in patients with solid cancer: a study protocol," BMJ Open, vol. 8, no. 8, Article ID e019502, 2018.

[40] C. Cheon and S. G. Ko, "Phase I study to evaluate the maximum tolerated dose of the combination of $\mathrm{SH} 003$ and docetaxel in patients with solid cancer: a study protocol," Medicine, vol. 99, no. 38, Article ID e22228, 2020.

[41] C. Cheon and S. G. Ko, "A phase I study to evaluate the safety of the herbal medicine SH003 in patients with solid cancer," Integrative Cancer Therapies, vol. 19, Article ID 1534735420911442, 2020.

[42] S. M. Abdel-Aziz, A. Aeron, and N. Garg, Microbes in Food and Health, p. 1, Springer, Berlin, Germany, 2016, online resource (X, 362 pages 331 illustrations, 321 illustrations in color.).

[43] W. Hsiao and L. Liu, "The role of traditional Chinese herbal medicines in cancer therapy - from TCM theory to mechanistic insights," Planta Medica, vol. 76, no. 11, pp. 1118-1131, 2010.

[44] K. T. Liou, C. Chen, N. Emard, K. A. Lynch, Y. N. Hou, and J. J. Mao, "Herbal topical analgesic for pain management: perspectives from cancer patients," Pain Medicine, vol. 22, 2021.

[45] J.-W. Lee, W. B. Lee, W. Kim, B.-I. Min, H. Lee, and S.-H. Cho, "Traditional herbal medicine for cancer pain: a systematic review and meta-analysis," Complementary Therapies in Medicine, vol. 23, no. 2, pp. 265-274, 2015.

[46] S. Yu, H. D. Peng, D. W. Ju et al., "Mechanisms of treatment of cancer pain with a topical Chinese herbal formula in rats," Chinese Medical Journal, vol. 122, no. 17, pp. 2027-2031, 2009.

[47] A. Nair and S. Jacob, "A simple practice guide for dose conversion between animals and human," Journal of Basic and Clinical Pharmacy, vol. 7, no. 2, pp. 27-31, 2016.

[48] P. Workman, E. O. Aboagye, E. O. Aboagye et al., "Guidelines for the welfare and use of animals in cancer research," British Journal of Cancer, vol. 102, no. 11, pp. 1555-1577, 2010.

[49] P. H. E. Hilkens, J. Verweij, C. J. Vecht, G. Stoter, and M. J. van den Bent, "Clinical characteristics of severe peripheral neuropathy induced by docetaxel (Taxotere)," Annals of Oncology, vol. 8, no. 2, pp. 187-190, 1997.

[50] I. Roglio, R. Bianchi, F. Camozzi et al., "Docetaxel-induced peripheral neuropathy: protective effects of dihydroprogesterone and progesterone in an experimental model," Journal of the Peripheral Nervous System, vol. 14, no. 1, pp. 36-44, 2009.

[51] C. L. Loprinzi, B. N. Reeves, S. R. Dakhil et al., "Natural history of paclitaxel-associated acute pain syndrome: prospective cohort study NCCTG N08C1," Journal of Clinical Oncology, vol. 29, no. 11, pp. 1472-1478, 2011.

[52] R. Asthana, L. Zhang, B. A. Wan et al., "Pain descriptors of taxane acute pain syndrome (TAPS) in breast cancer patientsa prospective clinical study," Supportive Care in Cancer, vol. 28, no. 2, pp. 589-598, 2020.

[53] J. Boyette-Davis and P. M. Dougherty, "Protection against oxaliplatin-induced mechanical hyperalgesia and intraepidermal nerve fiber loss by minocycline," Experimental Neurology, vol. 229, no. 2, pp. 353-357, 2011.

[54] C. Siau, W. Xiao, and G. Bennett, "Paclitaxel- and vincristineevoked painful peripheral neuropathies: loss of epidermal innervation and activation of Langerhans cells," Experimental Neurology, vol. 201, no. 2, pp. 507-514, 2006.

[55] J. Boyette-Davis, W. Xin, H. Zhang, and P. M. Dougherty, "Intraepidermal nerve fiber loss corresponds to the development of taxol-induced hyperalgesia and can be prevented by treatment with minocycline," Pain, vol. 152, no. 2, pp. 308-313, 2011.

[56] M. P. Giannoccaro, V. Donadio, C. Gomis Pèrez, W. Borsini, V. Di Stasi, and R. Liguori, "Somatic and autonomic small fiber neuropathy induced by bortezomib therapy: an immunofluorescence study," Neurological Sciences, vol. 32, no. 2, pp. 361-363, 2011.

[57] S. Wolf, D. Barton, L. Kottschade, A. Grothey, and C. Loprinzi, "Chemotherapy-induced peripheral neuropathy: prevention and treatment strategies," European Journal of Cancer, vol. 44, no. 11, pp. 1507-1515, 2008.

[58] M. I. Periquet, V. Novak, M. P. Collins et al., "Painful sensory neuropathy: prospective evaluation using skin biopsy," Neurology, vol. 53, no. 8, p. 1641, 1999.

[59] T. Krøigård, H. D. Schrøder, C. Qvortrup et al., "Characterization and diagnostic evaluation of chronic polyneuropathies induced by oxaliplatin and docetaxel comparing skin biopsy to quantitative sensory testing and nerve conduction studies," European Journal of Neurology, vol. 21, no. 4, pp. 623-629, 2014.

[60] W. Yang, K. Sung, F. Zhou et al., “Targeted mutation (R100W) of the gene encoding NGF leads to deficits in the peripheral sensory nervous system," Frontiers in Aging Neuroscience, vol. 10, p. 373, 2018.

[61] S. Geisler, R. A. Doan, A. Strickland, X. Huang, J. Milbrandt, and A. DiAntonio, "Prevention of vincristine-induced peripheral neuropathy by genetic deletion of SARM1 in mice," Brain: A Journal of Neurology, vol. 139, no. Pt 12, pp. 30923108, 2016.

[62] P. Marmiroli, G. Nicolini, M. Miloso, A. Scuteri, and G. Cavaletti, "The fundamental role of morphology in experimental neurotoxicology: the example of chemotherapyinduced peripheral neurotoxicity," Italian journal of anatomy and embryology, vol. 117, no. 2, pp. 75-97, 2012.

[63] S. L. Kyte, W. Toma, D. Bagdas et al., "Nicotine prevents and reverses paclitaxel-induced mechanical allodynia in a mouse model of CIPN," Journal of Pharmacology and Experimental Therapeutics, vol. 364, no. 1, pp. 110-119, 2018.

[64] H. Yousef, M. Alhajj, and S. Sharma, "Anatomy, skin (integument), epidermis," in StatPearls, StatPearls Publishing, Treasure Island, FL, USA, 2021.

[65] N. Mehmood, A. Hariz, S. Templeton, and N. Voelcker, "An improved flexible telemetry system to autonomously monitor 
sub-bandage pressure and wound moisture," Sensors, vol. 14, no. 11, pp. 21770-21790, 2014.

[66] A. J. McBain, C. A. O’Neill, and A. Oates, "Skin microbiology," Reference Module in Biomedical Sciences, pp. 734-747, Elsevier, Amsterdam, Netherlands, 2016.

[67] J. P. Cata, H. R. Weng, B. N. Lee, J. M. Reuben, and P. M. Dougherty, "Clinical and experimental findings in humans and animals with chemotherapy-induced peripheral neuropathy," Minerva Anestesiologica, vol. 72, no. 3, pp. 151-169, 2006.

[68] A. Muthuraman, N. Singh, and A. S. Jaggi, "Protective effect of Acorus calamus L. in rat model of vincristine induced painful neuropathy: an evidence of anti-inflammatory and anti-oxidative activity," Food and Chemical Toxicology, vol. 49, no. 10, pp. 2557-2563, 2011.

[69] N. Kiguchi, T. Maeda, Y. Kobayashi, T. Kondo, M. Ozaki, and S. Kishioka, "The critical role of invading peripheral macrophage-derived interleukin-6 in vincristine-induced mechanical allodynia in mice," European Journal of Pharmacology, vol. 592, no. 1-3, pp. 87-92, 2008.

[70] N. Kiguchi, T. Maeda, Y. Kobayashi, and S. Kishioka, "Upregulation of tumor necrosis factor-alpha in spinal cord contributes to vincristine-induced mechanical allodynia in mice," Neuroscience Letters, vol. 445, no. 2, pp. 140-143, 2008.

[71] Y.-Q. Zhou, Z. Liu, Z.-H. Liu et al., "Interleukin-6: an emerging regulator of pathological pain," Journal of Neuroinflammation, vol. 13, no. 1, p. 141, 2016.

[72] L. Leung and C. M. Cahill, "TNF- $\alpha$ and neuropathic pain - a review," Journal of Neuroinflammation, vol. 7, no. 1, p. 27, 2010.

[73] G. Fumagalli, L. Monza, G. Cavaletti, R. Rigolio, and C. Meregalli, "Neuroinflammatory process involved in different preclinical models of chemotherapy-induced peripheral neuropathy," Frontiers in Immunology, vol. 11, Article ID 626687, 2020.

[74] A. Saleh, S. K. Roy Chowdhury, D. R. Smith et al., "Diabetes impairs an interleukin- $\beta$-dependent pathway that enhances neurite outgrowth through JAK/STAT3 modulation of mitochondrial bioenergetics in adult sensory neurons," Molecular Brain, vol. 6, no. 1, p. 45, 2013.

[75] J. Hur, P. D. O’Brien, V. Nair et al., “Transcriptional networks of murine diabetic peripheral neuropathy and nephropathy: common and distinct gene expression patterns," Diabetologia, vol. 59, no. 6, pp. 1297-1306, 2016.

[76] V. Ganesh Yerra, G. Negi, S. S. Sharma, and A. Kumar, "Potential therapeutic effects of the simultaneous targeting of the Nrf2 and NF- $\kappa$ B pathways in diabetic neuropathy," Redox Biology, vol. 1, no. 1, pp. 394-397, 2013.

[77] S. V. Suryavanshi and Y. A. Kulkarni, "NF- $\kappa \beta$ : a potential target in the management of vascular complications of diabetes," Frontiers in Pharmacology, vol. 8, p. 798, 2017.

[78] K. Chopra and V. Tiwari, "Alcoholic neuropathy: possible mechanisms and future treatment possibilities," British Journal of Clinical Pharmacology, vol. 73, no. 3, pp. 348-362, 2012.

[79] F. T. Crews, C. J. Lawrimore, T. J. Walter, and L. G. Coleman, "The role of neuroimmune signaling in alcoholism," Neuropharmacology, vol. 122, pp. 56-73, 2017.

[80] J. M. Leger, S. Larue, and F. Dashi, "Dysimmune neuropathies: current diagnosis and therapy," Revue du Praticien, vol. 58, no. 17, pp. 1887-1889, 2008. 